\title{
Contrast-enhanced ultrasound (CEUS) of the lung reveals multiple areas of microthrombi in a COVID-19 patient
}

\author{
Alice Tee, Adrian Wong ${ }^{*}$ (D) Gibran Timothy Yusuf(D, Deepak Rao and Paul S. Sidhu(D)
}

๑ 2020 Springer-Verlag GmbH Germany, part of Springer Nature, corrected publication 2020

Dear Editor,

Peng et al. have outlined several characteristics common to all COVID-19 patients using lung ultrasound and the 12 zone method [1]. Zotzmann et al. in a follow-up letter raised the question on whether these areas of subpleural consolidation are actually indicative of segmental pulmonary embolus [2]. We would like to propose the use of contrast enhanced ultrasound (CEUS) to answer their question.

In order to assess the lungs for the presence of a thrombotic process, we used CEUS to evaluate the subpleural "consolidations" and they recognised as being typical to this disease. CEUS is a technique that involves the intravenous introduction of microbubbles consisting of a phospholipid shell surrounding a perflourocarbon gas (sulphur hexaflouride) the approximate size of red blood cells that can cross the capillary bed with transpulmonary stability. The result is a truly intravascular contrast agent, which can be detected using contrast specific modes on ultrasound. As a result, areas without contrast enhancement can be identified as being avascular.

We illustrate our findings with a case of a 61-year-old woman (see Fig. 1) with severe COVID-19 and a negative CTPA study. We were able show that these areas of irregularity, labelled as subpleural consolidation by Peng et al. were avascular and therefore most likely to represent 3-5 mm microinfarcts. Conversely, non-thrombotic consolidation would be seen to have some enhancement; a process not appreciated using other imaging modalities due to the superior spatial resolution of ultrasound.
Anecdotally, we have also seen these areas resolve with clinical improvement. It is becoming apparent that severe cases of COVID-19 are characterised by hyperinflammation and a thrombotic phenomenon. The theory of an underlying thrombotic process also corresponds with studies identifying admission D-dimer, prothrombin time and thrombocytopenia as prognostic markers [3]. Immuno-thrombosis is used to describe the interaction between platelets, coagulation factors and innate immune effector systems that, during an infection, results in secondary thrombus formation.

This is not the first time CEUS has been used to evaluate the presence of emboli. Trenker et al. found that, despite the lack of definite confirmation of PE on CT, peripheral subpleural consolidations with no or inhomogenous enhancement on CEUS to be highly suggestive of embolic consolidations [4]. A follow-up study by the same team used histological examination of six cases, and pulmonary infarction was found in all of them [5].

Post-mortem examinations of COVID-19 lungs support our theory but we believe this is the first time that this process has been demonstrated in the acute phase of the illness. We also hypothesise that this would also explain why high D-dimers have been linked to increased mortality [1].

We believe that our technique is readily generalisable given the widespread use of lung ultrasound in critical care and the fact that most radiology departments have the contrast agent. 


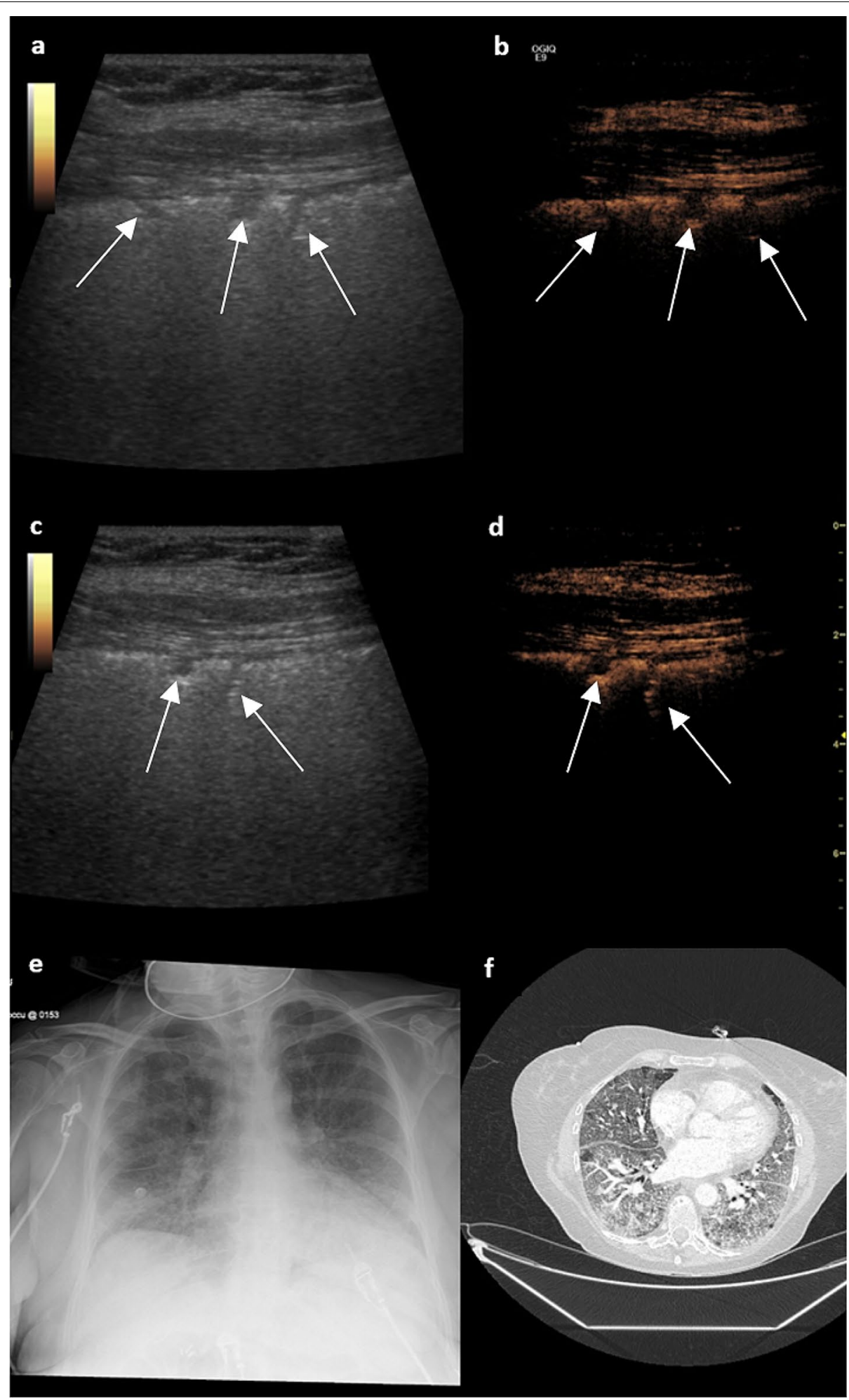

Fig. 1 a B mode lung ultrasound demonstrates subpleural consolidation (see arrows), a typical feature seen in COVID-19 pneumonia. b Simultaneous contrast enhanced lung ultrasound demonstrates non-enhancing subpleural areas which correspond to areas of infarction measuring just 3-5 mm, similar findings are present in all zones (c, d). e A chest radiograph demonstrates bilateral patchy peripheral infiltrates consistent with COVID-19 infection. f A CT PA shows signs typical for severe COVID-19 infection and no pulmonary embolism 


\section{Compliance with ethical standards}

\section{Conflicts of interest}

All the author's declare that they have no conflicts of interest.

\section{Publisher's Note}

Springer Nature remains neutral with regard to jurisdictional claims in published maps and institutional affiliations.

Accepted: 2 May 2020

Published online: 18 May 2020

\section{References}

1. Peng $Q$, Wang $X$, Zhang $L$ (2020) Findings of lung ultrasonography of novel corona virus pneumonia during the 2019-2020 epidemic. Intensive Care Med. https://doi.org/10.1007/s00134-020-05996-6
2. Zotzmann V, Lang CN, Bamberg F et al (2020) Are subpleural consolidations indicators for segmental pulmonary embolism in COVID-19? Intensive Care Med. https://doi.org/10.1007/s00134-020-06044-z

3. Henry BM, Vikse J, Benoit S, Favaloro EJ, Lippi G (2020) Hyperinflammation and derangement of renin-angiotensin-aldosterone system in COVID-19: A novel hypothesis for clinically suspected hypercoagulopathy and microvascular immunothrombosis, Clin Chim Acta. 507:167-173, https:// doi.org/10.1016/j.cca.2020.04.027.

4. Trenker C, Apitzsch JC, Pastor S et al (2017) Detection of peripheral embolic consolidations using contrast-enhanced ultrasonography in patients with no evidence of pulmonary embolism on computed tomography: A pilot study. J Clin Ultrasound JCU 45(9):575-579. https://doi. org/10.1002/jcu.22511

5. Trenker C, Dohse M, Ramaswamy A, Michel C, Görg C (2019) Histological validation of pulmonary infarction detected with contrast-enhanced ultrasound in patients with negative computed tomography pulmonary angiogram: a case series. J Clin Ultrasound JCU 47(8):461-465. https://doi. org/10.1002/jcu.22755 